\title{
Optimum Use of Selective Plated Media in Primary Processing of Respiratory Tract Specimens from Patients with Cystic Fibrosis
}

\author{
GARY V. DOERN* AND BRENDA BROGDEN-TORRES \\ Divisions of Clinical Microbiology and Infectious Diseases, University of \\ Massachusetts Medical Center, Worcester, Massachusetts 01655
}

Received 7 May 1992/Accepted 7 July 1992

\begin{abstract}
A total of 258 respiratory tract specimens from patients with cystic fibrosis were inoculated onto nine different plated media, and the rates of recovery of potential pathogens were compared. Media included sheep blood agar, enriched chocolate agar, MacConkey agar for gram-negative bacilli, chocolate agar containing bacitracin for Haemophilus spp., bromcresol green agar for yeasts, cetrimide agar for Pseudomonas spp., sheep blood agar containing colistin and nalidixic acid for gram-positive cocci, mannitol salt agar for Staphylococcus aureus, and oxidation-fermentation agar containing $300 \mathrm{U}$ of polymyxin $\mathrm{B}$ per $\mathrm{ml}$ and $2 \mathrm{U}$ of bacitracin per $\mathrm{ml}$ (OF-PBL medium) for Pseudomonas cepacia. With two exceptions, all of these media proved useful in recovering potential pathogens from respiratory tract specimens from patients with cystic fibrosis. The two exceptions were cetrimide agar and colistin-nalidixic acid-supplemented sheep blood agar, which were found to be superfluous. In addition, the results of this study further delineated the prevalence of selected bacteria and fungi in respiratory tract secretions from patients with cystic fibrosis. In rank order of frequency of isolation, we recovered isolates of Pseudomonas aeruginosa, Haemophilus parainfluenzae, Candida albicans, $S$. aureus, Haemophilus influenzae, molds, members of the family Enterobacteriaceae, yeasts other than Candida albicans, miscellaneous gram-negative bacilli, beta-hemolytic streptococci, $P$. cepacia, and Streptococcus pneumoniae.
\end{abstract}

Several unanswered questions surround the laboratory evaluation of respiratory tract secretions from patients with cystic fibrosis (CF). These include defining the optimum specimen for establishing the etiology of lower respiratory tract infections (i.e., pharyngeal swab versus deep throat swab versus expectorated sputum), as well as determining the best way to process specimens upon receipt in the laboratory (i.e., liquefaction versus direct plating and quantitative versus qualitative cultures) $(7-10,12)$. However, one issue for which agreement appears to exist concerns the need to use selective media for the primary processing of specimens from CF patients, irrespective of the nature of the specimen $(1-3,5,6$, $9,11,13-15)$. Selective media have been advocated for the optimum recovery of isolates of Haemophilus influenzae, Staphylococcus aureus, Pseudomonas aeruginosa, Pseudomonas cepacia, and other organisms. The intent of our investigation was to determine which of a variety of different media were of the greatest value in the routine processing of respiratory tract specimens from patients with CF.

A total of 258 consecutive specimens from patients known to have CF were included in this study. Among these, 157 specimens were throat swabs and the remaining 101 specimens were expectorated sputum specimens. The contents of throat swabs were eluted into $1.0-\mathrm{ml}$ volumes of Trypticase soy broth, and then approximately $0.1-\mathrm{ml}$ aliquots of these suspensions were transferred to the surfaces of primary plates with swabs and streaked for isolation. Expectorated sputum specimens were processed semiquantitatively after being liquefied. Briefly, equal aliquots of specimen and a solution containing $N$-acetyl-L-cystine and sodium citrate were mixed, vortexed for $60 \mathrm{~s}$, allowed to stand for $15 \mathrm{~min}$, and then mixed again. By

\footnotetext{
* Corresponding author.
}

using a swab, the contents of this suspension were transferred to the surfaces of culture plates and streaked for isolation.

Primary plates included 5\% sheep blood agar (BAP), enriched chocolate agar (CHOC), MacConkey agar (MAC), CHOC containing $300 \mu \mathrm{g}$ of bacitracin per ml (CHOC-BAC), bromcresol green agar (BCG), cetrimide agar (CET), 5\% BAP containing $10 \mu \mathrm{g}$ of colistin per $\mathrm{ml}$ and $10 \mu \mathrm{g}$ of nalidixic acid per ml (CNA), mannitol salt agar (MANN), and oxidationfermentation agar containing $300 \mathrm{U}$ of polymyxin $\mathrm{B}$ per $\mathrm{ml}$ and $2 \mathrm{U}$ of bacitracin per $\mathrm{ml}$ (OF-PBL). All plates were purchased from Remel Laboratories, Lenexa, Kans. BAP and CHOC were included as general-purpose media. The following selective media were used to facilitate the recovery of the following organisms: MAC, gram-negative bacilli, in particular members of the family Enterobacteriaceae; CNA, gram-positive cocci; CHOC-BAC, Haemophilus spp.; BCG, yeasts; CET, Pseudomonas spp.; MANN, $S$. aureus; and OF-PBL, $P$. cepacia.

All primary plates were incubated at $35^{\circ} \mathrm{C}$ in 5 to $7 \% \mathrm{CO}_{2}$ for 3 days and examined daily for the presence of growth. Viridans group streptococci, aerobic non-spore-forming gram-positive bacilli, species of staphylococci other than $S$. aureus, and Neisseria species other than $N$. meningitidis were characterized when present but were not included in the data analysis unless they had been isolated in pure culture in quantities of at least $3+$ (i.e., more than 30 colonies with growth into the third quadrant). All other organisms were identified to the species level by using conventional methods and criteria (1). When an individual species was recovered on more than one primary plate, a subjective assessment was made as to which one of the plates provided optimum growth (i.e., the largest number of well-isolated colonies) for the performance of identification procedures and susceptibility tests.

Among the 258 specimens included in this study, 2 yielded no growth and 8 yielded insignificant growth. The results of positive cultures with the remaining 248 specimens are sum- 


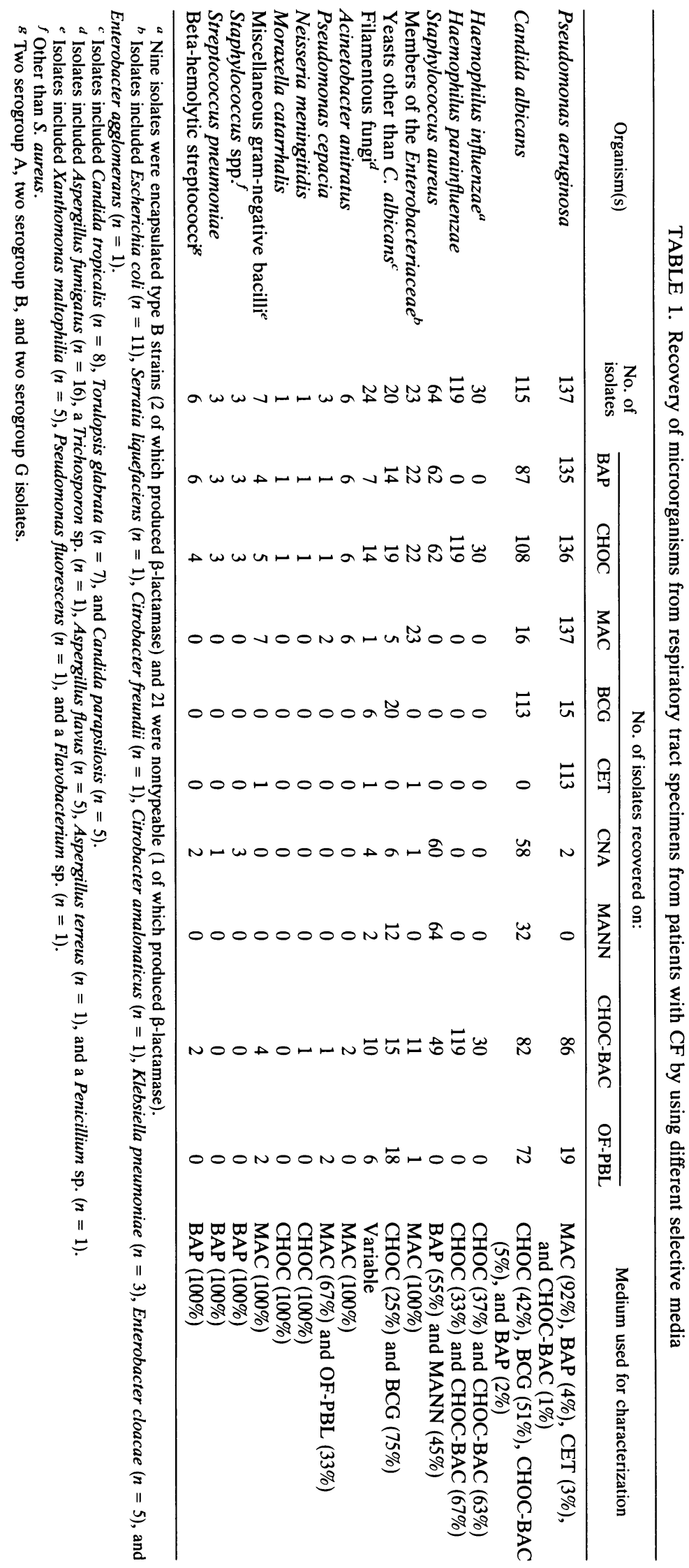


marized in Table 1. $P$. aeruginosa was recovered most frequently $(53.1 \%$ of specimens). Haemophilus parainfluen$z a e$ and Candida albicans were the next most common isolates (46.1 and $44.6 \%$ of specimens, respectively). $S$. aureus was recovered with intermediate frequency $(24.8 \%$ of specimens). $H$. influenzae (11.6\%), filamentous fungi $(9.3 \%)$, members of the Enterobacteriaceae $(8.9 \%)$, and yeasts other than $C$. albicans $(7.7 \%)$ were recovered less often but with similar frequency. All other bacteria, including Streptococcus pneumoniae, beta-hemolytic streptococci, $P$. cepacia, Acinetobacter calcoaceticus subsp. anitratus, and other miscellaneous gram-negative bacilli, were isolated only infrequently.

In most instances, selective media proved to be of value in processing respiratory tract specimens from patients with $\mathrm{CF}$ (Table 1). BCG yielded 133 of 135 isolates of yeast. The next most productive medium for yeasts was $\mathrm{CHOC}$, which yielded 127 of 135 isolates of yeast. In addition, BCG agar was judged to be the optimum medium for the performance of additional procedures, yielding $51 \%$ of the 115 isolates of $C$. albicans and all 20 of the isolates of yeast other than C. albicans. CHOCBAC was helpful in isolating and characterizing Haemophilus spp. All 30 isolates of $H$. influenzae and all 119 isolates of $H$. parainfluenzae were recovered on CHOC-BAC. This was also true for CHOC lacking bacitracin; however, in two-thirds of the cases, supplemental tests were performed with growth from the CHOC-BAC plates. This reflects the selective nature of CHOC-BAC for Haemophilus spp., with resultant isolated colonies and little overgrowth (4). MAC agar plates were equally useful for recovery and characterization of members of the Enterobacteriacae, $P$. aeruginos $a, A$. calcoaceticus subsp. anitratus, and other miscellaneous gram-negative bacilli (Table 1). MANN plates yielded all 64 isolates of $S$. aureus, and although 62 of these were also recovered on BAP and CHOC, growth from the MANN plate was used for isolate characterization in $45 \%$ of the cases.

In contrast, CET and CNA plates were of little value in processing respiratory tract specimens from patients with CF. CET plates yielded only 113 of $137(82.5 \%)$ isolates of $P$. aeruginosa and were the source of growth for additional tests in only $3 \%$ of the cases. As noted above, MAC plates were the most useful for the isolation and characterization of $P$. aeruginosa. Similarly, the rate of recovery of grampositive cocci other than $S$. aureus on CNA was only $50 \%$ (6 of 12), and in no case was growth from CNA plates used for additional studies. All 12 of these gram-positive cocci were isolated and then characterized from growth on BAP plates.

Unfortunately, the number of isolates of $P$. cepacia in the current study was too small to permit meaningful analyses of the utility of OF-PBL medium. It is clear from the literature, however, that optimum recovery of $P$. cepacia from CF patients requires the use of selective media $(2,3,5,11,13$, 15). Two different media, OF-PBL (13), as used in our investigation, and $P$. cepacia agar, have been most commonly employed (5). Two reports previously indicated that recovery rates with these two media are essentially comparable $(2,11)$.

The specimens included in the current study consisted of both throat swabs and expectorated sputa. There was no difference in the rates of recovery of individual organisms on the basis of specimen type, nor were there discernible differences in the relative yields of different media when analyzed according to specimen type.

Ascribing clinical significance to microorganisms recovered from respiratory tract secretions from $C F$ patients is often difficult, if not impossible. This is due to the high frequency with which the lower airways of asymptomatic CF patients become colonized with potential pathogens. For these rea- sons, it was not possible in the current study to analyze the results of cultures in light of their clinical significance.

In conclusion, the results of this investigation are consistent with the previous observations of others $(7,8,12)$ regarding those microorganisms most commonly recovered from respiratory tract secretions from CF patients. Furthermore, in general, the previous recommendations of others $(9,14)$ concerning the need for the use of several selective media for the optimum processing of CF specimens were confirmed. We found little value, however, in the routine use of CET and CNA plates. A useful battery of primary plates might include BAP, CHOC, MAC, BCG, MANN, CHOCBAC, and OF-PBL plates.

\section{REFERENCES}

1. Balows, A., W. J. Hausler, Jr., K. L. Herrmann, H. D. Isenberg, and H. J. Shadomy (ed.). 1991. Manual of clinical microbiology, 5th ed. American Society for Microbiology, Washington, D.C.

2. Black-Payne, C. B., M. B. Lierl, J. A. Bocchini, B. C. Hilman, E. D. Ramblin, and S. Silberman. 1987. Comparison of two selective media developed to isolate Pseudomonas cepacia from patients with cystic fibrosis. Diagn. Microbiol. Infect. Dis. 6:277-282.

3. Carson, L. A., O. C. Tablan, L. B. Cusick, W. R. Jarvis, M. S. Favero, and L. A. Bland. 1988. Comparative evaluation of selective media for isolation of Pseudomonas cepacia from cystic fibrosis patients and environmental sources. J. Clin. Microbiol. 26:20962100.

4. Chapin, K. C., and G. V. Doern. 1983. Selective media for recovery of Haemophilus influenzae from specimens contaminated with upper respiratory tract microbial flora. J. Clin. Microbiol. 17:1163-1165.

5. Gilligan, P. H., P. A. Gage, L. M. Bradshaw, D. V. Schidlow, and B. T. DeCicco. 1985. Isolation medium for the recovery of Pseudomonas cepacia from respiratory secretions of patients with cystic fibrosis. J. Clin. Microbiol. 22:5-8.

6. Gilligan, P. H., P. A. Gage, D. F. Welch, M. J. Muszynski, and K. R. Wait. 1987. Prevalence of thymidine-dependent Staphylococcus aureus in patients with cystic fibrosis. J. Clin. Microbiol. 25:1258-1261.

7. Hammerschlag, M. R. 1980. Bacteriology of sputum in cystic fibrosis: evaluation of dithiothreitol as a mucolytic agent. J. Clin. Microbiol. 11:552-557.

8. Iacocca, V. F., M. S. Sibinga, and G. J. Barbero. 1963. Respiratory tract bacteriology in cystic fibrosis. Am. J. Dis. Child. 106:115124.

9. Maduri-Tracyewski, M., C. L'Heureux, L. Escalona, A. Marcone, and D. Goldman. 1986. Facilitated detection of antibiotic resistant Pseudomonas in cystic fibrosis sputum using homogenized specimens and antibiotic containing media. Diagn. Microbiol. Infect. Dis. 5:299-305.

10. Parry, M. F., and H. C. Neu. 1977 . Effect of $\boldsymbol{n}$-acetylcysteine on antibiotic activity and bacterial growth in vitro. J. Clin. Microbiol. 5:58-61.

11. Tablan, O. C., L. A. Carson, L. B. Cusick, L. A. Bland, W. J. Martone, and W. R. Jarvis. 1987. Laboratory proficiency test results on use of selective media for isolating Pseudomonas cepacia from simulated sputum specimens of patients with cystic fibrosis. J. Clin. Microbiol. 25:485-487.

12. Welch, D. F. 1984. Clinical microbiology of cystic fibrosis. Clin. Microbiol. Newsl. 6:39-42.

13. Welch, D. F., M. J. Muszynski, C. H. Pai, M. J. Marcon, M. M. Hribar, P. H. Gilligan, J. H. Matsen, P. A. Ahlin, B. C. Hilman, and S. A. Chartrand. 1987. Selective and differential medium for recovery of Pseudomonas cepacia from the respiratory tracts of patients with cystic fibrosis. J. Clin. Microbiol. 25:1730-1734.

14. Wong, K., M. C. Roberts, M. Owens, M. Fife, and A. L. Smith. 1984. Selective media for the quantitation of bacteria in cystic fibrosis sputum. J. Med. Microbiol. 17:113-118.

15. Wu, B. J., and S. T. Thompson. 1984. Selective medium for Pseudomonas cepacia containing 9-chloro-9-(4-diethylaminophenyl)-10-phenylacridan and polymyxin B sulfate. Appl. Environ. Microbiol. 48:743-746. 